IV. Any phrase-equation whose members have like initial letters is interderivable with a phrase-equation whose members are free of parentheses and have like initial letters.

From the definition of the "terms" of a phrase, it is clear that if a phrase is free of parentheses all its terms are letters. Hence, by II, any phrase-equation $E$ whose members are free of parentheses is interderivable with any phrase-equation resulting from permutation of non-initial letters within members of $E$. Therefore any phrase-equation whose members are free of parentheses and have like initial letters is interderivable with an equation of canonical form. It then follows, by III and IV, that every phrase-equation is reducible to canonical form.

In view of $\S \S 3-4$, this concludes the proof that upon elimination of abbreviations all homogeneous linear identities with rational coefficients are generable by $(R)$ and $\left(R^{\prime}\right)$ from (A) and (B).

HARVARD UNIVERSITY

\title{
A CHARACTERISTIC PROPERTY OF SURFACES OF NEGATIVE CURVATURE $\dagger$
}

\section{BY E. F. BECKENBACH}

1. Introduction. Let there be given a piece of surface $S$ in a representation

(1) $S: \quad x=x(u, v), y=y(u, v), z=z(u, v), u^{2}+v^{2}<\rho^{2}$, with the following properties.

(a) $x(u, v), y(u, v), z(u, v)$ have continuous partial derivatives of the third order.

(b) The representation is isothermic; that is to say, $E=G$, $F=0$, where

$E=x_{u}{ }^{2}+y_{u}{ }^{2}+z_{u}{ }^{2}, F=x_{u} x_{v}+y_{u} y_{v}+z_{u} z_{v}, G=x_{v}{ }^{2}+y_{v}{ }^{2}+z_{v}{ }^{2}$,

the subscripts denoting differentiation.

We put $E=G=\lambda(u, v)$. Then $\lambda(u, v) \geqq 0$, and the representation is conformal except at points where $\lambda(u, v)=0$. The cus-

$\dagger$ Presented to the Society, April 7, 1934. 
tomary assumption of differential geometry, that $\lambda(u, v)>0$, is dropped because that assumption seems irrelevant to the present results.

Let the circle $\left(u-u_{0}\right)^{2}+\left(v-v_{0}\right)^{2} \leqq r^{2}$ in $u^{2}+v^{2}<\rho^{2}$ be denoted by $\left(u_{0}, v_{0} ; r\right)$; and let the area and length of boundary of the image on $S$ of $\left(u_{0}, v_{0} ; r\right)$ be denoted respectively by $a\left(u_{0}, v_{0} ; r\right)$ and $l\left(u_{0}, v_{0} ; r\right)$. On the above assumptions, the theorems expressed by the following formulas were proved first if $S$ lies on a plane, $\dagger$ then if $S$ is a minimal surface, $\ddagger$ and finally if $S$ is a surface of non-positive Gaussian curvature $K$ :

$$
\begin{aligned}
a\left(u_{0}, v_{0} ; r\right) & \geqq \pi r^{2} \lambda\left(u_{0}, v_{0}\right), \\
l\left(u_{0}, v_{0} ; r\right) & \geqq 2 \pi r\left[\lambda\left(u_{0}, v_{0}\right)\right]^{1 / 2} .
\end{aligned}
$$

Here it will be noted that $\lambda\left(u_{0}, v_{0}\right)$ and $\left[\lambda\left(u_{0}, v_{0}\right)\right]^{1 / 2}$ are respectively the area magnification ratio and the length magnification ratio at $\left(u_{0}, v_{0}\right)$.

Any representation of a $(u, v)$-domain $D$ on $S$ will be called a typical map provided conditions (a) and (b) are satisfied for that representation.

It is our purpose to demonstrate the extent to which these formulas (2) and (3) are characteristic of surfaces of negative Gaussian curvature $K$, namely, we shall show that (see $\$ 3$ ) there exist typical maps of $(u, v)$-domains $D$ on surfaces of positive curvature for which the above inequalities are satisfied for all $\left(u_{0}, v_{0} ; r\right)$ in $D$; but that (see $\left.\S 6\right)$ if either of these inequalities holds for all typical maps of $(u, v)$-domains $D$ on $S$ and for all $\left(u_{0}, v_{0} ; r\right)$ in $D$, then $K \leqq 0$ on $S . \|$

The above will follow immediately from the simple lemma (see $\S 4,5):$ A necessary and sufficient condition that the Gaussian curvature $K$ of a surface $S$, which admits of typical representations, be $\leqq 0$ wherever $K$ is defined on $S$, is that for all typical maps of $(u, v)$-domains on $S$, the function $\lambda(u, v)$ be subharmonic.

$\dagger$ L. Bieberbach, Zur Theorie und Praxis der konformen Abbildung, Palermo Rendiconti, vol. 38 (1914), pp. 98-112.

$\ddagger \mathrm{E}$. F. Beckenbach, The area and boundary of minimal surfaces, Annals of Mathematics, vol. 33 (1932), pp. 658-664.

$\S \mathrm{E}$. F. Beckenbach and T. Radó, Subharmonic functions and surfaces of negative curvature, Transactions of this Society, vol. 35 (1933), pp. 662-674.

$\|$ That is, $K \leqq 0$ wherever $K$ is defined on $S$, namely at all points for which $\lambda(u, v)>0$. 
2. Lemma. Let $S$ be a surface given in a representation

$$
S: \quad x=x(u, v), y=y(u, v), z=z(u, v),
$$

$(u, v)$ in some domain $D$ (not necessarily simply-connected), the coordinate functions having continuous first partial derivatives satisfying $E=G=\lambda(u, v), F=0$. If $\lambda(u, v)$ is subharmonic in $D$, then $a\left(u_{0}, v_{0} ; r\right) /\left(\pi r^{2}\right)$ is a convex function of $\log r$ in any circular disc lying in $D$. Or if $[\lambda(u, v)]^{1 / 2}$ is subharmonic in $D$, then $\dagger$ $l\left(u_{0}, v_{0} ; r\right) /(2 \pi r)$ is a convex function of $\log r$ in any circular annulus lying in $D$.

This lemma merely expresses special cases of a fundamental property of the linear-mean and of the area-mean of subharmonic functions. $\ddagger$

Thus

$$
\frac{l\left(u_{0}, v_{0} ; r\right)}{2 \pi r}=\frac{1}{2 \pi} \int_{0}^{2 \pi}\left[\lambda\left(u_{0}+r \cos \phi, v_{0}+r \sin \phi\right)\right]^{1 / 2} d \phi
$$

is the linear-mean of $[\lambda(u, v)]^{1 / 2}$, and

$$
\frac{a\left(u_{0}, v_{0} ; r\right)}{\pi r^{2}}=\frac{1}{\pi r^{2}} \iint_{\xi^{2}+\eta^{2}<r^{2}} \lambda\left(u_{0}+\xi, v_{0}+\eta\right) d \xi d \eta
$$

is the area-mean of $\lambda(u, v)$.

For any point $\left(u_{0}, v_{0}\right)$ of $D$, we define, for $r=0$,

$$
\begin{aligned}
& {\left[\frac{a\left(u_{0}, v_{0} ; r\right)}{\pi r^{2}}\right]_{r=0}=\lim _{r=0} \frac{a\left(u_{0}, v_{0} ; r\right)}{\pi r^{2}}=\lambda\left(u_{0}, v_{0}\right),} \\
& {\left[\frac{l\left(u_{0}, v_{0} ; r\right)}{2 \pi r}\right]_{r=0}=\lim _{r=0} \frac{l\left(u_{0}, v_{0} ; r\right)}{2 \pi r}=\left[\lambda\left(u_{0}, v_{0}\right)\right]^{1 / 2} .}
\end{aligned}
$$

We have then the immediate corollaries, which hold as long as the circular disc $\left(u_{0}, v_{0} ; r\right)$ remains in $D$ :

$\dagger$ See C. H. Dix, The length of closed level curves of a harmonic function, this Bulletin, vol. 39 (1933), pp. 24-25.

$\ddagger$ F. Riesz, Über subharmonische Funktionen und ihre Rolle in der Funktionentheorie und in der Potentialtheorie, Acta Szeged, vol. 2 (1924-26), pp. 87100 , and Sur les fonctions subharmoniques et leur rapport a la théorie du potentiel, Acta Mathematica, vol. 48 (1926), pp. 329-343. For the generalization to the area-mean, see P. Montel, Sur les fonctions convexes et les fonctions sousharmoniques, Journal de Mathématiques, (9), vol. 7 (1928), pp. 29-60. 
If $\lambda(u, v)$ is subharmonic in $D$, then

$$
a\left(u_{0}, v_{0} ; r\right) /\left(\pi r^{2}\right)
$$

is a non-decreasing function of $r$;

$$
a\left(u_{0}, v_{0} ; r\right) \geqq \pi r^{2} \lambda\left(u_{0}, v_{0}\right) .
$$

If $[\lambda(u, v)]^{1 / 2}$ is subharmonic in $D$, then

$$
l\left(u_{0}, v_{0} ; r\right) /(2 \pi r)
$$

is a non-decreasing function of $r ; \dagger$

$$
l\left(u_{0}, v_{0} ; r\right) \geqq 2 \pi r\left[\lambda\left(u_{0}, v_{0}\right]^{1 / 2} .\right.
$$

(A.1) and (B.1) follow from the fact that a function which is convex out to $-\infty$, and is bounded, necessarily is non-decreasing. (A.2) and (B.2) are immediate corollaries of (A.1) and (B.1), respectively.

If in the above we replace the word "subharmonic" by "superharmonic," the lemma still holds, with "convex" replaced by "concave." And the four corollaries hold, with "non-decreasing" replaced by "non-increasing" and with the signs of inequality reversed.

3. Example. The equations $\ddagger$

$$
x=\frac{2 u}{u^{2}+v^{2}+1}, \quad y=\frac{2 v}{u^{2}+v^{2}+1}, \quad z=\frac{u^{2}+v^{2}-1}{u^{2}+v^{2}+1}
$$

give the familiar stereographic projection of the unit sphere $(K \equiv+1)$ with center $(0,0,0)$ in the $(x, y, z)$-space on the $(u, v)$-plane, the $u$ - and $v$-axes coinciding, respectively, with those of $x$ and $y$.

As is well known, this representation is isothermic; indeed, computations yield

$$
E=G=\lambda(u, v)=\frac{4}{\left(u^{2}+v^{2}+1\right)^{2}}, F=0 .
$$

Further computations give the following expressions for the Laplacians $\Delta \lambda \equiv \lambda_{u u}+\lambda_{v v}$ and $\Delta\left(\lambda^{1 / 2}\right) \equiv\left(\lambda^{1 / 2}\right)_{u u}+\left(\lambda^{1 / 2}\right)_{v v}$ :

$\dagger$ A fortiori, then, $l\left(u_{0}, v_{0} ; r\right)$ is a non-decreasing function of $r$; see E. F. Beckenbach and T. Rado, loc. cit.

‡ See, for instance, L. R. Ford, A utomorphic Functions, 1929, p. 120. 


$$
\begin{aligned}
\Delta \lambda & =\frac{32}{\left(u^{2}+v^{2}+1\right)^{4}}\left(2 u^{2}+2 v^{2}-1\right), \\
\Delta\left(\lambda^{1 / 2}\right) & =\frac{8}{\left(u^{2}+v^{2}+1\right)^{3}}\left(u^{2}+v^{2}-1\right) .
\end{aligned}
$$

Since a necessary and sufficient condition that a function $g(u, v)$ which admits of continuous second derivatives be subharmonic (superharmonic) is that $\Delta g$ be $\geqq 0(\leqq 0), \dagger$ it follows that $\lambda(u, v)$ is subharmonic for $u^{2}+v^{2}>1 / 2$ and superharmonic for $u^{2}+v^{2}<1 / 2$, and that $[\lambda(u, v)]^{1 / 2}$ is subharmonic for $u^{2}+v^{2}>1$ and superharmonic for $u^{2}+v^{2}<1$.

It follows (see \$2) that (2) holds provided the circle $\left(u_{0}, v_{0} ; r\right)$ lies with its interior in $u^{2}+v^{2} \geqq 1 / 2$, but that the sign of inequality is reversed if the circle lies in $u^{2}+v^{2} \leqq 1 / 2$. A similar statement holds for (3), according as the circle lies with its interior in $u^{2}+v^{2} \geqq 1$ or in $u^{2}+v^{2} \leqq 1$.

Thus, whether or not $\lambda(u, v)$ for a small piece $S^{*}$ of the spherical surface is subharmonic, and whether or not (2) or (3) holds on $S^{*}$, depend not solely on the nature of the surface but also, in this example, on the choice of typical parameters.

4. THEOREM 1. If $K \leqq 0$ wherever $K$ is defined on a surface $S$ which admits of typical representations, then for any typical representation of $a(u, v)$-domain on $S$, the function $\lambda(u, v)$ is of class $P L, \ddagger$ and therefore $[\lambda(u, v)]^{1 / 2}$ and $\lambda(u, v)$ are subharmonic.

For surfaces in typical representation, $E=G=\lambda(u, v), F=0$, the Gaussian curvature $K$ is given, at points where $K$ is defined, by the expression: $\S$

$$
K=\frac{1}{2 \lambda^{3}}\left(\lambda_{u}^{2}+\lambda_{v}^{2}-\lambda \Delta \lambda\right)=-\frac{1}{2 \lambda} \Delta \log \lambda .
$$

† See F. Riesz, loc. cit.

$\ddagger$ A function $p(u, v)$, defined in a domain $D$, is said to be of class $P L$ in $D$ provided $p(u, v)$ is continuous and $\geqq 0$ in $D$, and $\log p(u, v)$ is subharmonic in the part of $D$ where $p(u, v)>0$. See E. F. Beckenbach and T. Rad6, Subharmonic functions and minimal surfaces, Transactions of this Society, vol. 35 (1933), pp. 648-661.

$\S$ See, for instance, E. F. Beckenbach and T. Rad6, loc. cit.; the results of this section are taken from that source. 
It is seen here that $K \leqq 0$ is equivalent to $\Delta \log \lambda \geqq 0$; consequently, $\lambda(u, v)$ is of class $P L$, and therefore, a fortiori, $[\lambda(u, v)]^{1 / 2}$ and $\lambda(u, v)$ are subharmonic. Or directly, if it is desired to show only that $[\lambda(u, v)]^{1 / 2}$ is subharmonic, we note first that at points $\left(u_{0}, v_{0}\right)$ where $\lambda=0$, it follows from $\lambda^{1 / 2} \geqq 0$, that $\lambda^{1 / 2}$ satisfies the sub-mean property

$$
\left[\lambda\left(u_{0}, v_{0}\right)\right]^{1 / 2} \leqq \frac{1}{2 \pi} \int_{0}^{2 \pi}\left[\lambda\left(u_{0}+r \cos \phi, v_{0}+r \sin \phi\right)\right]^{1 / 2} d \phi,
$$

and secondly that for other points, $K \leqq 0$ and (4) imply

$$
\Delta\left(\lambda^{1 / 2}\right)=\frac{1}{2} \lambda^{-3 / 2}\left[\lambda \Delta \lambda-\frac{1}{2}\left(\lambda_{u}^{2}+\lambda_{v}^{2}\right)\right] \geqq 0 ;
$$

consequently, $[\lambda(u, v)]^{1 / 2}$ is subharmonic. A similar direct discussion might be given for $\lambda(u, v)$.

It follows (see \$2) that for any typical representation of a $(u, v)$-domain $D$ on a surface $S$ for which $K \leqq 0$ wherever $K$ is defined on $S$, the inequalities (2) and (3) hold for all $\left(u_{0}, v_{0} ; r\right)$ in $D$.

Conversely, (4) shows that if $\lambda(u, v)$ is of class $P L$ for one typical representation on $S$, then $K \leqq 0$ wherever $K$ is defined on $S$; consequently, $\lambda(u, v)$ is of class $P L$, and therefore subharmonic, for all typical representations of $(u, v)$-domains on $S$.

The example of $\$ 3$ shows, on the other hand, that $\lambda(u, v)$ being subharmonic for one typical representation on a surface $S$ does not imply $K \leqq 0$ on $S$. Nevertheless, we have the following result.

5. TheORem 2. If for all typical representations of $(u, v)$-domains on the surface $S$ given by (1), the area magnification ratio $\lambda(u, v)$ is a subharmonic function of $u$ and $v$, then the Gaussian curvature $K$ of $S$ is $\leqq 0$ wherever $K$ is defined on $S$.

With $S$ given in the representation (1), fix $\left(u_{0}, v_{0}\right)$, any point in $u^{2}+v^{2}<\rho^{2}$; we shall show, on the above assumption, that $K \leqq 0$ at the point on $S$ corresponding to $\left(u_{0}, v_{0}\right)$, provided $K$ is defined there. Since $u^{2}+v^{2}<\rho^{2}$ can be mapped conformally on itself, any interior point being carried to the origin, and since this mapping induces a new typical representation of $u^{2}+v^{2}<\rho^{2}$ on $S$, there is no loss of generality in assuming $\left(u_{0}, v_{0}\right)=(0,0)$. Again, since a rotation about the origin gives a conformal map 
of the circle on itself, we may assume that the $v$-axis is tangent, at $(0,0)$, to a contour line for the function $\lambda(u, v)$, so that

$$
\lambda_{v}(0,0)=0 \text {. }
$$

Let an $(\alpha, \beta)$-domain $D$ be mapped conformally on $u^{2}+v^{2}<\rho^{2}$ by the analytic function

$$
u+i v=f(\alpha+i \beta) .
$$

This mapping induces a typical representation of $D$ on $S$, for which the area magnification ratio is given by

$$
\mu(\alpha, \beta)=x_{\alpha}^{2}+y_{\alpha}^{2}+z_{\alpha}^{2}=\lambda(u, v)\left|f^{\prime}(\alpha+i \beta)\right|^{2} .
$$

In (6) we substitute the particular function

$$
u+i v=(\alpha+i \beta)^{m}-2 \rho,
$$

where $m$ is a real parameter different from zero. This function maps the angle $|\phi|<\pi /(2|m|)$, where $\alpha+i \beta=r e^{i \phi}$, on the right-hand half-plane $u>-2 \rho$, and consequently maps some domain $D$ in the angle conformally on the circle $u^{2}+v^{2}<\rho^{2}$. Of course, $D$ might not lie on a single-sheeted plane, for $D$ might wind about the origin; but $D$ remains away from the origin and is free of branch-points.

For the function (7), we have

$$
\begin{gathered}
u=r^{m} \cos m \phi-2 \rho, v=r^{m} \sin m \phi \\
\mu(\alpha, \beta)=\lambda(u, v) m^{2} r^{2 m-2} .
\end{gathered}
$$

A computation shows that the Laplacian

$$
\Delta \mu=\mu_{r r}+\frac{\mu_{r}}{r}+\frac{\mu_{\phi \phi}}{r^{2}}
$$

is given by

$$
\begin{aligned}
\Delta \mu=m^{4} r^{2 m-4}\left\{4 \lambda\left(\frac{m-1}{m}\right)^{2}\right. & +4 \lambda_{u} r^{m}\left(\frac{m-1}{m}\right) \cos m \phi \\
& \left.+4 \lambda_{v} r^{m}\left(\frac{m-1}{m}\right) \sin m \phi+r^{2 m} \Delta \lambda\right\} .
\end{aligned}
$$

By assumption, $\Delta \mu \geqq 0$ for all $m \neq 0$ and for all $(\alpha, \beta)$ in $D$, so that the quantity in brackets is $\geqq 0$ for $(u, v)$ in $u^{2}+v^{2}<\rho^{2}$. In particular, at $(u, v)=(0,0)$, we have $\phi=0$ and $r^{m}=2 \rho$, so 
that, writing $k$ for $(m-1) / m$, we get at $(u, v)=(0,0)$ the quadratic inequality in $k$

$$
\lambda(0,0) k^{2}+2 \lambda_{u}(0,0) \rho k+\rho^{2} \Delta \lambda(0,0) \geqq 0
$$

for all $k \neq 1$, and therefore for all $k$. Necessary conditions for this are that $\Delta \lambda(0,0) \geqq 0$ and that the discriminant of the quadratic be $\leqq 0$ :

$$
\left[\lambda_{u}(0,0)\right]^{2}-\lambda(0,0) \Delta \lambda(0,0) \leqq 0 .
$$

It follows from (4), (5) and (8) that if $\lambda(0,0)>0$, so that $K$ is defined at the point on $S$ corresponding to $(u, v)=(0,0)$, then $K \leqq 0$ there.

6. Conclusion. Inequalities (2) and (3), written as

$$
\begin{gathered}
\lambda\left(u_{0}, v_{0}\right) \leqq \frac{1}{\pi r^{2}} \iint_{\xi^{2}+\eta^{2}<r^{2}} \lambda\left(u_{0}+\xi, v_{0}+\eta\right) d \xi d \eta, \\
{\left[\lambda\left(u_{0}, v_{0}\right)\right]^{1 / 2} \leqq \frac{1}{2 \pi} \int_{0}^{2 \pi}\left[\lambda\left(u_{0}+r \cos \phi, v_{0}+r \sin \phi\right]^{1 / 2} d \phi,\right.}
\end{gathered}
$$

express defining properties* of subharmonic functions. Consequently if, for a certain typical representation on a surface $S$ of a $(u, v)$-domain $D,(2)$ or (3) holds for all $\left(u_{0}, v_{0} ; r\right)$ in $D$, then for that representation $\lambda(u, v)$ or $[\lambda(u, v)]^{1 / 2}$, as the case might be, is subharmonic. In either case, $\lambda(u, v)$ is subharmonic for that representation, since $[\lambda(u, v)]^{1 / 2}$ being subharmonic implies the same for $\lambda(u, v)$.

By the result of $\$ 5$, then, if either (2) or (3) holds for all typical representations on $S$ of $(u, v)$-domains $D$ and for all $\left(u_{0}, v_{0} ; r\right)$ in $D$, so that $\lambda(u, v)$ is subharmonic for all typical representations, it follows that $K \leqq 0$ wherever $K$ is defined on $S$. And conversely, as we have pointed out (see $\$ 4$ ), if $S$ is a surface given in typical representation, for which $K \leqq 0$ whereever $K$ is defined on $S$, then (2) and (3) hold for all $\left(u_{0}, v_{0} ; r\right)$ in the domain of definition. To this extent, then, the inequalities (2) and (3) are characteristic of surfaces of negative curvature.

The Rice Institute

* See J. E. Littlewood, On the definition of a subharmonic function, London Mathematical Society Journal, vol. 2 (1927), pp. 189-192. 